The INL is a

U.S. Department of Energy

National Laboratory

operated by

Battelle Energy Alliance

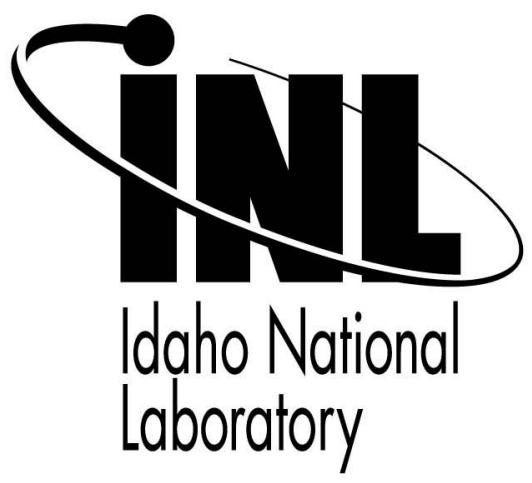

\title{
Improving Emergency Response and Human- Robotic Performance
}

\section{Joint Meeting and Conference of the Institute of Electrical and Electronics Engineers (IEEE) and Human Performance / Root Cause / Trending / Operating Experience / and Self Assessment (HPRCT)}

\author{
David I. Gertman \\ David J. Bruemmer \\ R. Scott Hartley
}

This is a preprint of a paper intended for publication in a journal or proceedings. Since changes may be made before publication, this preprint should not be cited or reproduced without permission of the author. This document was prepared as an account of work sponsored by an agency of the United States Government. Neither the United States Government nor any agency thereof, or any of their employees, makes any warranty, expressed or implied, or assumes any legal liability or responsibility for any third party's use, or the results of such use, of any information, apparatus, product or process disclosed in this report, or represents that its use by such third party would not infringe privately owned rights. The views expressed in this paper are not necessarily those of the United States Government or the sponsoring agency. 


\title{
Improving Emergency Response and Human-Robotic Performance
}

\author{
David I. Gertman, David J. Bruemmer, and R. Scott Hartley \\ Human Factors and I\&C Systems Department, Idaho National Laboratory, Idaho Falls, ID, USA. \\ \{david.gertman, david.bruemmer, and r.hartley, @inl.gov\}
}

\begin{abstract}
Preparedness for chemical, biological, and radiological/nuclear incidents at nuclear power plants (NPPs) includes the deployment of well trained emergency response teams. While teams are expected to do well, data from other domains suggests that the timeliness and accuracy associated with incident response can be improved through collaborative human-robotic interaction. Many incident response scenarios call for multiple, complex procedure-based activities performed by personnel wearing cumbersome personal protective equipment (PPE) and operating under high levels of stress and workload. While robotic assistance is postulated to reduce workload and exposure, limitations associated with communications and the robot's ability to act independently have served to limit reliability and reduce our potential to exploit human -robotic interaction and efficacy of response. Recent work at the Idaho National Laboroaty (INL) on expanding robot capability has the potential to improve human-system response during disaster management and recovery. Specifically, increasing the range of higher level robot behaviors such as autonomous navigation and mapping, evolving new abstractions for sensor and control data, and developing metaphors for operator control have the potential to improve state-of-the-art in incident response. This paper discusses these issues and reports on experiments underway intelligence residing on the robot to enhance emergency response.
\end{abstract}

\section{INTRODUCTION}

The INL is taking steps to design robotic capabilities that can be used to address a variety of radiological hazards in nuclear power plants. We begin with a brief overview of how the nuclear power industry currently addresses these hazards and then introduce new technologies that can be used to augment the current toolset of capabilities available to emergency response decision makers. Finally, we discuss the first in a series of live experiments which will use humans and robots together in a simulated response to radiological hazards. The goal is to better understand the benefits and limitations to using existing teleoperated robotic approaches as well as newly available autonomous and intelligent robotic capabilities.
In 1996, the US Nuclear Regulatory Commission (NRC) published in the Federal Register the Federal Radiological Emergency Response Plan (FRERP) Operational Plan covering a concept of operations for responding to radiological emergencies. The FRERP covers "any peace time radiological emergency that has actual, potential, or perceived radiological consequences within the United States." The level of response is a function of the type and amount of material involved, location of the emergency, and impact or potential impact on the public and the environment. The plan goes on to inform that emergencies at nuclear facilities or during the transportation of radioactive materials fall within the scope of the plan. Some 16 Federal Agencies including the US NRC participate in the FRERP.

(http://www.fas.org/nuke/guide/usa/doctrine/national/fre rp.htm).

Guidance called out in the FRERP presents coordination by Federal Agencies including the role of the Agencies with or without State request. The authority for response is Executive Order (E.O.) 12241, National Contingency Plan, September 29, 1980. "This E.O. delegates to the Director of Federal Emergency Mangement Administration (FEMA) the responsibility for publishing the National Contingency Plan (i.e., the FRERP) for accidents at nuclear power facilities and requires that it be published from time to time in the Federal Register. Executive Order 12241 has been amended by Executive Order 12657, FEMA Assistance in Emergency Preparedness Planning at Commercial NuclearPowerPlants."

(http://www.fas.org/nuke/guide/usa/doctrine/national/fre $\underline{\text { rp.htm }}$

The Nuclear Energy Institute (NEI) January 2007 states that NRC requirements for nuclear power plants call for emergency response plan training:

"All U.S. nuclear plants must participate in reviewed, full-scale emergency exercises every two years. For each exercise, the utility creates a confidential emergency scenario for use by plant staff and local emergency response organizations, including law enforcement, local hospitals, radiological monitoring teams and others. Post-exercise critiques by the federal agencies and exercise participants identify areas for correction in future exercises or any improvements needed in the plan itself. 
The NRC also requires that plants conduct training drills in alternate years to test their emergency response capabilities for dealing with a range of events. State and local emergency management officials often participate in these drills. Since the drills are not graded, the NRC permits supervised instruction and resolution of the drill scenarios' problems. NRC inspectors at the plants often observe the drills. After the drills, plant officials incorporate lessons learned and corrective actions into the emergency response plan.

$\mathrm{NRC}$ headquarters and regional staff participate in at least one emergency exercise per year in each of the four regions. The agency's emergency response facility and teams are critiqued on their responses to the simulated emergency."

http://www.nei.org/doc.asp?catnum=3\&catid=291

The constitution of the response team including decisions whether or not to utilize robots to reduce exposure, and improve reliability associated with mapping is decided on a local level. This decision is often made without a great deal of hard data to inform the decision makers regarding the benefits and limitations of various approaches and technologies. Quite simply, this information is not available because the studies have not yet been performed. Work is now being performed at the INL to develop robotic solutions and apply these to specific emergency response experiments. The purpose is to better inform decision makers of the performance tradeoffs associated with the use of various robotic technologies and approaches.

The behaviors required of personnel (or of personnel in conjunction with one or more robots) in response to incidents are roughly the same, whether the situation is the result of an intentional act or an accident. These behaviors can include stabilizing emergency conditions, preparing characterization of radiological conditions and immediate consequences, and development of a monitoring plan. "The coordinated response to contain or mitigate a threatened or actual release of radioactive material would be essentially the same whether it resulted from an accidental or deliberate act. Malevolent acts involving improvised nuclear or radiation dispersal devices, may complicate the magnitude of the threat and the need for specialized technical expertise/actions. Therefore, sabotage and terrorism are not treated as separate types of emergencies.

\section{Role of Department of Energy (DOE).}

The Department of Energy (DOE) owns and operates a variety of radiological activities throughout the United States. These activities at fixed nuclear sites include the use, storage, and shipment of a variety of radioactive materials the shipment of spent reactor fuel the production, assembly, and shipment of nuclear weapons and special nuclear materials the production and shipment of radioactive sources for space ventures and the storage and shipment of radioactive and mixed waste. DOE is responsible for the safe operation of these activities and should an emergency occur at one of its sites or an activity under its control, DOE will be the lead Federal Agency for the Federal response.

Due to its technical capabilities and resources, the DOE may perform other roles within the Federal response to a radiological emergency. With extensive, field-based radiological resources throughout the United States available for emergency deployment, the DOE responds to requests for offsite radiological monitoring and assessment assistance and serves as the initial coordinator of all such Federal assistance (to include initial management of the FRMAC) to State and local governments. With other specialized, deployable assets, DOE assists other Federal agencies responding to malevolent nuclear emergencies, accidents involving nuclear weapons not under DOE custody, emergencies caused by satellites containing radioactive sources, and other radiological incidents as appropriate:

For these reasons, having the capabilities to simulate human behaviors in a wide-array of novel or untested environmental context is essential in planning and developing human factors design interfaces for next generation control room to ensure plant safety.

DOE has trained personnel, radiological instruments, mobile laboratories, and radioanalytical facilities located at its national laboratories, production, and other facilities throughout the country. Through eight Regional Coordinating Offices, these resources form the basis for the Radiological Assistance Program (RAP), which can provide technical assistance in any radiological emergency. DOE can provide specialized radiation detection instruments and support for both its response as lead Federal Agency and as initial coordinator of Federal radiological monitoring and assessment assistance. Some of the specialized resources and capabilities include

1. "Aerial monitoring capability for tracking dispersion of radioactive material and mapping ground contamination

2. A computer-based, emergency preparedness and response predictive capability that provides rapid predictions of the transport, diffusion, and deposition of radionuclides released to the atmosphere and dose projections to people and the environment

3. Specialized equipment and instruments and response teams for locating radioactive materials and handling damaged nuclear weapons 
4. Medical experts on radiation effects and the treatment of exposed or contaminated patients and

5. Support facilities for DOE response, including command post supplies, communications systems, generators, and portable video and photographic capabilities."

Currently, there is no established performance requirement directing the use of robots as part of emergency response planning. Extrapolating laboratory findings to the field have a number of associated challenges. The INL has used robots to address radiological hazards for two decades. Over the last five years, particular emphasis has been placed on developing intelligent and semi-autonomous robotic solutions Also; much has been learned by applying robot behaviors to other areas such as countermine operations and urban search and rescue (USAR). Although, experiments have demonstrated the potential value of autonomy for various hazardous environments, the mainstream involvement of robots in many civilian applications remains a promise unfulfilled.

Yanco (2004) and Burke (2004) contend that there are a number of challenges present in real world operations that have slowed the acceptance of robots, particularly in the area of search and rescue and remote radiation characterization. They both point out that situation awareness and poor communication remain problematic. We believe that an approach embracing mixed initiative control is the logical answer to this dilemma, particularly in instances where the operator's view of the robot world is naturally constrained. The INL seeks to find an answer in a combination of 3-D visualization, improved sensor capability, enhanced robot intelligence including spatial reasoning, enhanced robot initiative and improved metaphors for control.

\section{STATE OF ART AND HUMAN ROBOTIC COLLABORATION}

Robots have a distinct advantage in a variety of settings including those where human access is difficult, impractical or dangerous. For a review of those instances see for example, Murphy (2004). However, the ground rules for the nature of the partnership between robots and people are still being written. The degree to which people are comfortable with levels of robot autonomy varies as a function of the application and environment. In extreme emergency situations including those we address in this paper and in our research, the trust the human has and their knowledge of robot activities figures prominently. This includes communication functions and performing tasks to strict performance requirements. In the case of urban search and rescue, countermine operations, or emergency response to a dirty bomb, the operator needs to be confident that performance requirements are being met.

In order to support research in emergency response, the INL has developed a graded approach to autonomous behavior and engineered the robot intelligence kernel $\left(\mathrm{RIK}^{\mathrm{TM}}\right)$.[ref] In performing this work we have taken the approach that the intelligence should reside on the robot itself and that, additionally, the robot should be capable of protecting itself and performing a variety of intrinsic functions without human intervention. We have implemented a number of behaviors including guarded motion, obstacle avoidance, mapping and localization, path planning and waypoint navigation

To accomplish these behaviors, perceptual algorithms running on the robot fuse a variety of range sensor information. For instance, on a robot used for USAR applications, a laser range finder is mounted on the front, and 17 sonar are located around the mid-section of the robot. The robot also has highly sensitive bump strips in the rear and in the rear and front that register if anything has been touched. To protect the top of the robot, especially the cameras, we have also added an array of infrared proximity sensors that indicate when an object is less than nine inches from the robot. Additional infrared proximity sensors have been placed on the bottom of the robot and point ahead of the robot towards the ground in order to prevent the robot from traveling into open space (e.g. traveling off of a landing down a stairway). Together these sensors provide a field of protection around the robot and allow the operator to command the robot with full confidence.

A variety of robotic capabilities may be needed across different emergency scenarios. The RIK can be placed on many different robots including robots with drastically different drive trains, geometries, and sensor suites. The portability and re-configurability of the RIK insures that the same basic behaviors and interface options are available to the user regardless of the robot platform. This is also useful for reducing training time of personnel since a single operator control unit (OCU) can be used to operate many different kinds of robots.

In recent discussions with explosive ordinance disposal (EOD) experts form the $22^{\text {nd }}$ Chemical Battalion stationed at Pine Bluff Arsenal, in Arkansas, the issue of communications was very high priority. Current robotic control schemes involve significant communications from and to the robot for directional control and visual feedback. To permit deployment within shielded structures or caves, we have developed a customized communication protocol, which allows very low bandwidth communications to pass over a serial radio link only when needed. The interface itself 
then unfolds these simple packets into a comprehensive interface. Although our visual link and wireless Ethernet link were subject to dropouts during a recent competition, the $900 \mathrm{Mhz}$ data link that we used to transmit this protocol suffered no data loss throughout the entire competition and exhibition.

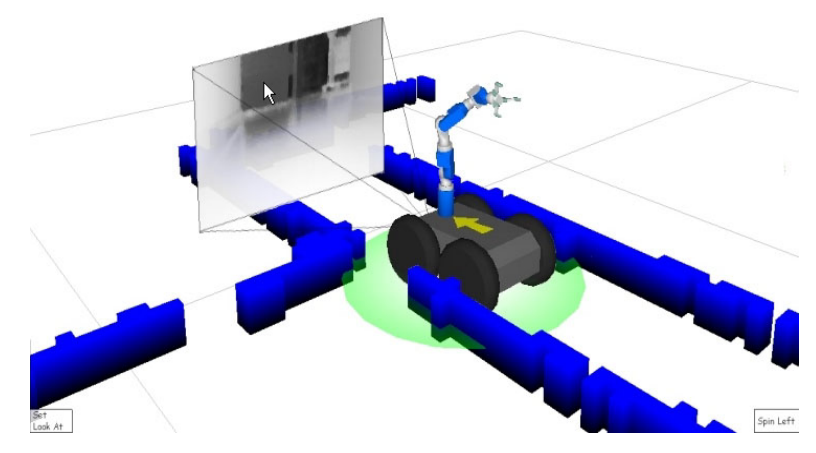

III. AN INTEGRATED PERFORMANCE MODELING APPROACH

As part of the information gathering process supporting our studies, users defined issues experienced while addressing radiological emergencies. Their experiences were drawn from their time of service in Iraq, but those given below can be generalized to other radiological emergencies:

Use of protective equipment. There is an inherent difficulty of operations involving the use of hazardous materials (HAZMAT) personnel protective equipment (PPE) gear. This is scenario specific, however, in a chemical, biological, or radiological scenario it is likely that personnel would wear multiple layers of gloves, full hooded suits, and respirators or supplied air. The results can include severely compromised peripheral vision and limited dexterity making task execution "exponentially more difficult" in terms of time and effort. Additionally, many operators may be needed, depending on the difficulty of the task and time required. There may be multiple operators, multiple teams (no less than six trained personnel at a time) and support personnel for decontamination and wash down. This can be greatly reduced when a robot is deployed.

Heat factors. High temperatures pose a significant constraint for personnel wearing HAZMAT suits and responding to a dirty bomb. Personnel may have to enter the facility wearing full protective gear including a self contained breathing apparatus (SCBA), and a heart rate monitor. A typical stay time for SCBA use is approximately $1 / 2$ hour. Tethered air may extend stay times. High temperatures may preclude entry without cooling vests or other cooling measures. There is no stay time requirement for the robot analog.
Communication Challenges. Challenges associated with fiber optic exist because because tethered communications cause problems when robots are close together. Cables and reels increase system weight and the robots can run them over or get them tangled, especially when backing up to avoid an obstacle. They said that take-up reels are sometimes used. These considerations complicate robot operations making control of multiple robots difficult and requiring a high level of skill.

Information presentation. Regarding information presentation, experts replied that "Colors don't matter, but a key to aid deciphering must be provided. Further, you want a 2 milli-radiation exposure man (mrem) line (press) and a 10 mrem line (personnel safety boundary) should be indicated. This could be present in the form of a contours line notifying the operator that personnel time in that zone must be limited. These factors and findings from previous studies went into the design of the study described below. Findings from this study are the subject of a separate report to be published at a later date.

\section{Evaluating Collaborative Behavior}

Purpose: The study plan aims to rigorously evaluate the effectiveness and suitability of control capabilities and interfaces with three degrees of robotic autonomy for unmanned ground vehicles (UGVs) ranging from teleoperated with no autonomy, similar to current activities, to mostly autonomous with an advanced operator control unit. The experiment is designed to assess human/robot collaboration and effectiveness in mapping, detecting, communicating, determining, and marking the location of a simulated dirty bomb.

Robot control will be conducted from a mobile trailer set up outside of a building that is part of the critical infrastructure test range (CITRC) (Figure 1). Two radiation sources will be placed inside the building, one in a relatively open (easy) area and the other in a cluttered office (difficult). Subjects will not enter the building with the radiological sources exposed or be exposed to radiation above background levels. A radiation control technician (RCT) will provide continuous monitoring when radiological sources are exposed. An experiment controller will be stationed in the trailer to begin the experiment, answer questions, simulate military-type controls (hold points), and protect equipment.

For each of three modes, sources will be placed and exposed and the robot will enter the facility through one of the entry doors. The subject will be directed to search 
the building using the specified equipment and capabilities to find and mark the location of the sources on the floor plan. Subjects will be advised that there is to be equal importance associated with logging the radiation levels and with pinpointing the source locations and communicating. They will also be instructed to record radiation levels on the floor plan. Procedures will require that the robot maintain a distance of 2 feet from the source. Once both sources are identified and the area searched, the robot will be driven to a marked position and directed to exit the building quickly. Once the robot has exited the building, the experimental session is concluded. The duration of each session is expected to be one hour or less and the building may be subdivided to reduce the area subject to search.

The CITRC includes an airfield and 5 facilities covering several miles with roads, buildings, water, sewer, communications and electrical distribution systems.

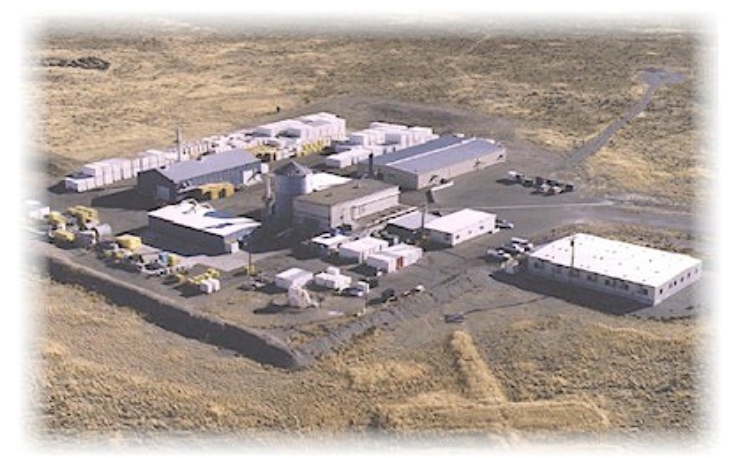

All subjects will be trained and allowed to operate robots in all three modes prior to the experiment. Each subject will be assessed in each mode, with presentation order being randomized.. The equipment for each of the 3 modes is as follows.

Mode 1 - Basic Tele-operation - The equipment for Mode 1 includes:

iRobot ${ }^{\mathrm{TM}}$ Packbot

Floor plan

Log paper/notepad

MGP Instruments AMP-50 Gamma detector (dual)

Detector output will include level in counts and audible clicks

900 MHZ Freewave transceiver for control communications

802.11 b wireless transmitter for video output

Screen for display of video images

A joystick or gamepad-type controller as for Sony Playstation
Mode 2 - Tele-operation with mapping and guarded motion - The equipment for Mode 2 includes:

iRobot ${ }^{\mathrm{TM}}$ Packbot

Floor plan

Log paper/notepad

MGP Instruments AMP-50 Gamma detector (dual)

Detector output will include level in counts and audible clicks

900 MHZ Freewave transceiver for control communications

802.11 b wireless transmitter for video output

Robot intelligence kernel (RIK)

Laser range finder

On-The-Fly mapping and localization for building interior features

Radiation sensor reading abstraction (plume data represented on map

Robot will employ obstacle avoidance behaviors

Screen for display of video and abstracted map and sensor images

A joystick or gamepad-type controller as for Sony Playstation

Mode 3 - Collaborative tasking - The equipment for Mode 3 includes:

iRobot $^{\text {TM }}$ Packbot

Floor plan

Log paper/notepad

MGP Instruments AMP-50 Gamma detector (dual)

Detector output will include level in counts and audible clicks

900 MHZ Freewave transceiver for control communications

802.11 b wireless transmitter for video output

Robot intelligence kernel (RIK)

Laser range finder

On-The-Fly mapping and localization for building interior features

Radiation sensor reading abstraction (plume data represented on map)

Robot will include all robotic autonomy behaviors

Screen for display of video and abstracted map and sensor images

Advanced operator control unit (OCU) with simplified controls including target mode

A joystick or gamepad-type controller as for Sony Playstation

Range of behavior and relationship to presentation mode In the collaborative tasking condition the robot is capable of path planning, obstacle avoidance, Go To, target, and radiation painting.. The human behaviors 
are to observe and to take action by dropping and clicking on a target. The robot finds its own path to the target while gathering information. In the guarded motion condition the robot capability includes guarded motion, mapping and radiation painting. In the basic teleoperation, video, guarded motion is used to stop the robot from running into obstacles and a time penalty is invoked. In both the guarded motion and basic tele operation conditions, the human takes action by driving a joystick.

From this study we hope to attain some basic information on the value of a robot, value of mapping and the value of increased levels of autonomy. Dependent measures include localization accuracy, logging accuracy, trespass on exclusion zones, and time to negotiate obstacles.

\section{B. Designing the interface}

Obviously, one of the keys to superior collaborative performance lies in the design of the control interface. Moving from using a joystick to drive the robot to moving a target and allowing the robot to find its way requires both a different perspective on collaboration as well as a difference in the interface. Many other improvements have gone into the design of the interface. This work includes review of data fusion methods, improvements to controllers i.e., smaller and lighter, modification of interface software and design of data representation schemes. Also, new to this study is the onboard incorporation of additional advanced HAZMAT sensors such as radiation sensors.

As part of our design approach, we try to reduce complexity for the operator while increasing their understanding of the environment. We do this by imparting intelligence to the robot and simplifying the interface as much as possible. The underlying algorithms may be complex but the operator's view of the robots world need not be complex. Arguments for the benefits of employing simplicity in design abound. For recent thoughts on the topic see Maeda (2006). One of our principle design guidelines is to review, review and review. The final design is as much as result of the failures as it is successes. Previous studies at the INL have indicated without the right interaction metaphor, users do not know what to expect from their mysterious and complex robot "peers." Increased autonomy and initiative is open to review in the sense that autonomy may actually complicate the task and frustrate the operator if the interface and underlying interaction metaphor are not designed appropriately. The robot must demonstrate reliable predictable behavior if it is to be trusted by the operator.

\section{SUMMARY / CONCLUSION}

In emergency response, personnel are often placed at risk from environmental conditions. Heat, radiation, and chemical spills can reduce the stay time from personnel thus requiring redundancy in terms of personnel. Some of the tasking can be shifted to the robot, reducing the required number of personnel and personnel exposure. We believe that the use of robots can result in reduced exposure, fewer personnel, and less operator fatigue. An experiment is proposed and discussed that will also empirically assess the benefits in terms of quality of data, task time till completion, and subjective measures such as operator confidence.

The goal of our research is to have the operator view the robot as a team mate as opposed to a taxi with a sensor suite on board. We recognize that the robot is a tool, but we believe that this tool can be trusted to perform a variety of task sub-components with a high degree of proficiency - which may exceed a human performing the same task. These subtasks include mapping and localization, obstacle avoidance, path planning and creating a dynamic representation of the radiation plume. The use of 3-D imaging based on our collaborations with SRI Company and Brigham Young University (BYU) add a great improvement to the interface. Replacing the "joystick" metaphor with alternate control designs and improved operator representations of the world offers opportunity to expand the ways in which robots are tasked. We recognize that different robots have different uses and advantages and have designed the intelligence kernel so that it can be implemented on various robot platforms. To this end, a number of near term exploratory studies are planned. This paper has provided a synopsis of the experiment plan for the first in a series of studies focused on emergency response to radiological hazards. We hope that this new robotic capability as well as the increased understanding achieved by these studies will benefit the nuclear power plant industry as well as DOE. Because the application of collaborative behavior in high risk emergency response situations can serve to reduce exposure and potential loss of life, progress in this area can not come fast enough.

\section{DISCLAIMER}

This paper was prepared as an account of work sponsored by an agency of the United States Government. Neither the United States Government nor any agency thereof, nor any of their employees, makes any warranty, expressed or implied, or assumes any 
legal liability or responsibility for any third party's use, or the results of such use, of any information, apparatus, product, or process disclosed in this paper, or represents that its use by such third party would not infringe privately owned rights.

\section{REFERENCES}

[1] (http://www.bt.cdc.gov/radiation) Centers for Disease Control (CDC) Radiation Emergencies. Accessed May 2007.

[2] (http://www.fas.org/nuke/guide/usa/doctrine/national/frerp.htm Nuclear Regulatory Commission (NRC) Rederal Radiological Emergency Response Plan (FRERP) - Operational Plan. Published May 1996. Accessed May 2007.

[3] http:/hazmat.dot.gov/regs/overhml.pdf, Federal Hazardous Transportation Law, (HMR; 49 CFR Parts 171-180),

[4] http://www.nei.org/doc.asp?catnum $=3 \&$ catid $=291$

Nuclear Energy Institute (NEI), January 2007. Accessed May 2007.

[5] J Drury, Hestand, D., and H. Yanco Design guidelines for improved human robotic interaction, in Proceddings of $\mathrm{CHI}$, 2004, April 24-29 Vienna, Austria. ACM1-58113-703$6 / 04 / 2004$

[6] D.J. Bruemmer, D.A. Few, S. Sirin, H. Hunting, M. Walton, and F. Carney. Autonomous robot for sensor characterization. Robotics and Remote Systems for Hazardous Environments, Gainesville, Florida, March 28-31, 2004.

[7] Bruemmer, D. J., Dudenhoeffer, D., and Marble, J. Dynamic Autonomy for Urban Search and Rescue, AAI Mobile Robot Competetion, 2002: 33-37.

[8] Yanco, H. A., \& Drury, J. (2004a). Where Am I? Acquiring Situation Awareness Using a Remote Robot Platform. Proceedings of the Institute of Electrical and Electronics Engineers (IEEE) Conference on Systems, Man and Cybernetics, 2835-2840

[9] Burke, J. L., Murphy, R R., Coovert, M. D., \& Riddle, D. L. (2004a). Moonlight in Miami: A field study of human-robot interaction in the context of an urban search and rescue disaster response training exercise. Human-Computer Interaction, 19, 85-116

[10] Murphy, R. R. (2004). Human-robot interaction in rescue robotics. Institute of Electrical and Electronics Engineers (IEEE) Transactions on Systems, Man, and Cybernetics-Part C, $34(2), 138-153$

[11] Maeda, J. (2006) The Laws of Simplicity: Design, Technolgoy, Business,Life, the MIT Press, Cambridge, MA. 